\title{
Exaggerated relative nasal-temporal asymmetry of macular capillary blood flow in patients with clinically significant diabetic macular oedema
}

\author{
C Hudson, J G Flanagan, G S Turner, H C Chen, M H Rawii, D McLeod
}

Br J Ophthalmol 2005;89:142-146. doi: 10.1136/bjo.2003.037317

\begin{abstract}
Aims: To compare the macular capillary blood flow of patients with clinically significant diabetic macular oedema (DMO) with that of non-diabetic subjects and to determine the relation between blood flow and capillary leakage in patients with DMO.

Methods: The sample comprised 45 non-diabetic subjects (mean age 59 years) and 18 type 2 patients with clinically significant DMO (mean age 60 years). Macular capillary blood flow measurements were acquired using the Heidelberg retina flowmeter (HRF) and a $10^{\circ} \times 2.5^{\circ}$ scan field centred on the fovea. Fluorescein angiography was undertaken on each of the diabetic patients after the completion of HRF measurements.

Results: Temporal macular capillary blood flow was significantly lower for the patients with clinically significant DMO compared with age matched non-diabetic subjects (ANCOVA, $p=0.0011$ ) while relative nasal-temporal asymmetry of macular capillary blood flow was significantly higher $(p=0.0125)$. Nasal-temporal asymmetry of macular capillary blood flow was significantly higher for the patients with DMO and capillary leakage within the scan area (two tailed $t$ test, $p=0.0071$ ). Macular capillary blood flow was always lower in areas of DMO and capillary leakage.

Conclusion: Capillary blood flow was reduced in areas of DMO and capillary leakage, suggesting the presence of a localised perturbation of capillary blood flow regulation.
\end{abstract}

R etinal capillaries lack an autonomic nerve supply but they are thought to have an intrinsic ability to constrict or dilate-that is, autoregulate, in order to ensure adequate blood flow to retinal tissues under changing conditions. ${ }^{1}$ In addition, autoregulation is thought to be primarily facilitated by the vascular reactivity of upstream arterioles. Disturbance of retinal capillary blood flow is a feature of many ocular diseases, including diabetic retinopathy and glaucoma. A number of non-invasive instruments have been designed to measure retinal haemodynamics. ${ }^{2-5}$ Scanning laser Doppler flowmetry (SLDF-that is, the combination of a laser Doppler flowmeter and a scanning laser system) is unique in that it provides a two dimensional quantifiable perfusion map of retinal capillary blood flow rather than a measurement of flow at a single point. ${ }^{6}$ SLDF has potential as a clinical tool to non-invasively visualise and quantify retinal capillary blood flow. ${ }^{6-8}$

Retinal capillary blood flow disturbance is fundamental to the pathogenesis of diabetic retinopathy. ${ }^{19-14}$ However, retinal blood flow as measured in the major retinal vessels of patients with diabetic retinopathy is reported by some to be reduced $^{9-11}$ and by others to be increased ${ }^{12-14}$ when compared to non-diabetic subjects, in part depending on the severity of the retinopathy or on the heterogeneity of the group. This study focuses on diabetic patients with diabetic macular oedema (DMO) and on the macular capillary circulation. Macular oedema is the leading cause of visual impairment and legal blindness in people with diabetes. ${ }^{15}$ To the best of our knowledge, no studies have investigated the impact of DMO on SLDF derived measurements of macular capillary blood flow. The objectives of the study were: firstly, to compare the macular capillary blood flow of patients with clinically significant DMO to that of non-diabetic subjects; secondly, to investigate the relation between macular capillary blood flow measured using SLDF and capillary leakage assessed using fundus fluorescein angiography (FFA) in patients with clinically significant DMO.

\section{PATIENTS AND METHODS \\ Sample}

The sample comprised 45 non-diabetic subjects and 18 patients with clinically significant DMO. The sample size was calculated by referencing unpublished data from patients with DMO and non-diabetic controls. A difference in macular capillary blood flow between patients with DMO and nondiabetic subjects of 100 arbitrary units was found (that is, the expected effect size, $E$ ) and the group mean standard deviation (SD) for the patient group was 95 units. The standardised effect size, $E / S D$, for the SLDF procedure was 1.05. To test the hypotheses with a two tailed $\alpha=0.05$ and a $\beta=90 \%$ (that is, power), the required sample size for the group of patients with DMO was 18. The non-diabetic subject group included 18 males and 27 females of mean age 59 years (SD 9 years, range $45-77$ years). The DMO group included 12 males and six females of mean age 60 years (SD 7 years, range $45-75$ years) and mean diabetes diagnosis of 8 years (range $2-18$ years). All patients were type 2 diabetics. Two were insulin controlled and 16 were receiving oral hypoglycaemic medication. Three diabetic patients had heart disease, another patient had renal disease, two had experienced stroke, and six patients were taking medication to control systemic hypertension (four patients were taking angiotensin converting enzyme inhibitors for systemic hypertension while another two patients were taking calcium channel blockers). Four diabetic patients had abnormally elevated serum creatinine levels and six patients had elevated serum urea levels (three of these six patients exhibited abnormal elevation of both creatinine and urea). None of the non-diabetic subjects had diagnosed heart disease, or renal disease, or stroke and only three subjects were taking medication to control systemic hypertension.

Abbreviations: DMO, diabetic macular oedema; FAZ, foveal avascular zone; FFA, fundus fluorescein angiography; HRF, Heidelberg retina flowmeter; SLDF, scanning laser Doppler flowmetry 
One eye of each volunteer was randomly assigned to the study, assuming both eyes met the appropriate inclusion criteria. Twenty three right eyes and 22 left eyes were selected for the non-diabetic group while for the DMO group eight right eyes and 10 left eyes were included. All patients with diabetes exhibited clinically significant DMO as defined by the Early Treatment Diabetic Retinopathy Study ${ }^{16}$ criteria, $^{2}$ specifically retinal thickening within $500 \mu \mathrm{m}$ of the fovea (with or without some non-circinate exudates). There was a general absence of changes associated with ischaemic maculopathy, including obvious foveal avascular zone abnormalities (as confirmed by the independent assessment of the fluorescein angiograms undertaken by two of the medical retina specialists). Two medical retina specialists independently confirmed the diagnosis of clinically significant DMO using stereo fundus biomicroscopy before inclusion of the patient into the study. In addition, all volunteers were required to achieve a logMAR visual acuity of 0.25 or better, using the $96 \%$ contrast Regan chart (that is, Snellen equivalent visual acuity of 6/10). Informed consent was obtained from all volunteers following an explanation of the nature of the procedures and the study gained the approval of the Central Manchester research ethics committee.

Exclusion criteria comprised (i) a distance refractive error greater than plus or minus 6.00 dioptres sphere and/or more than plus or minus 1.50 dioptres cylinder; (ii) a family history of glaucoma in a first degree relative; (iii) a family history of diabetes in a first degree relative (applicable to the normal group only); (iv) an intraocular pressure $\geqslant 22 \mathrm{~mm} \mathrm{Hg}$; (v) any other eye disease or disorder (including lenticular opacity); (vi) any previous laser photocoagulation treatment; and (vii) proliferative diabetic retinopathy and its sequelae-for example, vitreous haemorrhage and retinal detachment. Eyes with the following Lens Opacities Classification System (LOCS) III $^{17}$ grades were excluded from the study: (i) nuclear colour > NC2; (ii) nuclear opalescence $>\mathrm{NO} 2$; (iii) cortical cataract $>\mathrm{C} 2$; and (iv) posterior subcapsular cataract $\geqslant \mathrm{Pl}$.

\section{Procedures}

All volunteers were dilated using $1 \%$ Mydriacyl before imaging.

\section{Scanning laser Doppler flowmetry}

The Heidelberg retina flowmeter (HRF, software version 1.02) uses a $780 \mathrm{~nm}$ wavelength infrared diode laser to measure the intensity, and thereby the Doppler shift, of back scattered light. The instrument has a spatial resolution of approximately $10 \mu \mathrm{m}$ and a depth resolution 300-400 $\mu \mathrm{m}$. The laser scanning system of the HRF enables the measurement of a $10^{\circ}$ horizontal $\times 2.5^{\circ}$ vertical (that is, approximately $2.7 \mathrm{~mm} \times 0.7 \mathrm{~mm}$ ) field with a resolution of 256 pixels $\times 64$ lines, respectively. Each line of 256 pixels is scanned 128 times at a repetition rate of $4000 \mathrm{~Hz}$. The resulting image acquisition time is 2.048 seconds. ${ }^{5}$ The underlying theory of the HRF has been explained in detail elsewhere. ${ }^{5} 18$

Volunteers viewed a fixation light at 6 metres through a periscope with the non-study eye-that is, permitting the patient to see around the housing of the HRF and thereby maintain fixation during imaging of the macula. The focus setting of the HRF was adjusted to distinguish the nerve fibre layer striations of each individual and to produce maximum image brightness (care was taken to avoid overexposure of pixels). Corneal curvature and refractive error were entered into the HRF database prior to image acquisition. A $10^{\circ} \times 2.5^{\circ}$ (that is, $2.7 \mathrm{~mm} \times 0.7 \mathrm{~mm}$ ) scan field was employed. Seven consecutive HRF images were acquired, centred on the fovea, for each volunteer by a single operator $(\mathrm{CH})$.

\section{Fluorescein angiography}

FFA was undertaken on each of the patients with clinically significant DMO after the completion of HRF measurements. "Optimal" early and late phase angiograms were selected on the basis of the agreement of the three ophthalmologists involved in the study (DM, GST, and HCC). Consequently, the times of early and late phase angiograms varied between patients. Photographs of the early and late phase angiograms were printed using a $15 \times 11.5 \mathrm{~cm}$ format, and areas of capillary leakage and ischaemia were mapped onto acetate sheets overlying the angiogram photographs. Mapping of the capillary leakage and ischaemia was undertaken by two of the ophthalmologists (GST and HCC). Each of the ophthalmologists was masked to the FFA assessment of the other. The group of patients with clinically significant DMO was then subdivided into those patients without DMO and capillary leakage within the HRF scan area $(n=13)$ and those with DMO and capillary leakage within the scan area $(\mathrm{n}=5)$.

\section{Analysis}

Automatic full field perfusion image analysis (AFFPIA, version 3.3.0) was undertaken on the aligned HRF images since it reduced measurement artefact. ${ }^{19}$ For non-diabetic subjects, and for patients without capillary leakage within the HRF scan area, measurements were undertaken for a $2.5^{\circ}$ diameter circle centred on the fovea and for the complete temporal and nasal macular areas either side of the circle. For diabetic patients with capillary leakage within the SLDF scan area, measurements were undertaken for a $2.5^{\circ}$ diameter circle centred on the fovea and for mirror image circles (minimum $1^{\circ}$ diameter) either side of the fovea; one of the circles was centred on an area of capillary leakage.

A repeated measures analysis of co-variance (re-ANCOVA) was used to establish the significance of differences in macular capillary blood flow between the two groups. Volunteers were age matched between the two groups-that is, the re-ANCOVA utilised 18 non-diabetic subjects and 18 age matched patients with clinically significant DMO. The reANCOVA was undertaken on foveal, temporal, nasal, and nasal-temporal asymmetry (that is, the absolute difference in flow for the selected measurement areas either side of the fovea) of macular capillary blood flow parameters. Group was the between subjects factor, macular capillary blood flow was the dependent variable, and age was the covariate.

Two tailed $t$ tests (taking into account the unequal sample sizes of the two groups) were undertaken to compare the temporal macular capillary blood flow and the nasaltemporal asymmetry of macular capillary blood flow between the patients with and without DMO and capillary leakage within the scan area. In addition, 95\% confidence limits for nasal, foveal, temporal, and relative nasal-temporal asymmetry of macular capillary blood flow were determined from the data of the non-diabetic group. The blood flow values of each individual patient with DMO (that is, the mean of seven SLDF readings) were compared to the $95 \%$ confidence limits of the non-diabetic group.

\section{RESULTS}

The group mean results for all the non-diabetic subjects and for the patients with clinically significant DMO of macular capillary blood flow are detailed in table 1. The age matched re-ANCOVA demonstrated that temporal macular capillary blood flow was significantly lower for the patients with clinically significant DMO $(\mathrm{p}=0.0011)$. Nasal-temporal asymmetry of macular capillary blood flow was significantly higher for the patients with clinically significant DMO $(\mathrm{p}=0.0125)$. There was no statistically significant difference in the nasal $(p=0.2643)$ or foveal $(p=0.2904)$ macular 
Table 1 Group mean capillary blood flow (arbitrary units) for the nasal, foveal and temporal SLDF scan areas, and nasal-temporal asymmetry of SLDF macular blood flow, for non-diabetic subjects $(n=45)$ and for patients $(n=18)$ with clinically significant diabetic macular oedema (DMO)

\begin{tabular}{lrrrr}
\hline & Nasal & Fovea & Temporal & Asymmetry \\
\hline Non-diabetic subjects & & & & \\
Mean & 218 & 161 & 221 & 65 \\
SD & 103 & 91 & 97 & 35 \\
Min & 51 & 34 & 45 & 12 \\
Max & 504 & 430 & 491 & 61 \\
Median & 200 & 146 & 201 & 123 \\
Patients with DMO & & & 122 & 75 \\
Mean & 159 & 111 & 71 & 21 \\
SD & 129 & 93 & 22 & 332 \\
Min & 57 & 17 & 265 & 112 \\
Max & 553 & 394 & 118 & \\
Median & 81 & & \\
\hline
\end{tabular}

capillary blood flow between the non-diabetic subjects and the patients with clinically significant DMO. It should be noted that the magnitude of the group variances was high with the standard deviation typically being approximately $50 \%$ of the group mean.

The two tailed $t$ tests indicated that temporal macular capillary blood flow was not significantly different between the patients with and without DMO and capillary leakage within the scan area $(p=0.1989)$. However, nasal-temporal asymmetry of macular capillary blood flow was significantly higher for the patients with DMO and capillary leakage within the scan area $(p=0.0071)$ compared with the patients without.
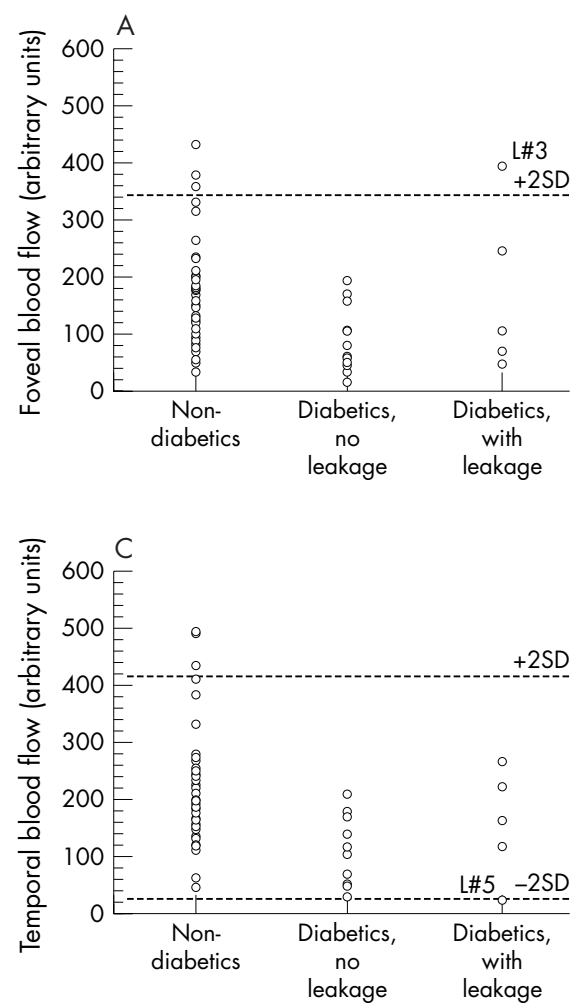

Scatter plots of individual mean (that is, of seven scans) nasal, foveal, and temporal macular capillary blood flow, and nasal-temporal asymmetry of macular capillary blood flow for the non-diabetic subjects and for the patients with clinically significant DMO (subdivided into those with and without DMO and capillary leakage within the scan area) are shown in figure 1. One patient with clinically significant DMO within the HRF scan area exhibited nasal and foveal macular capillary blood flow values that were higher than the normal 95\% confidence limits (that is, L\#3) - the patient did not exhibit DMO, as indicated by fluorescein leakage, in the nasal or foveal areas of the HRF scan. One patient with clinically significant DMO within the HRF scan area
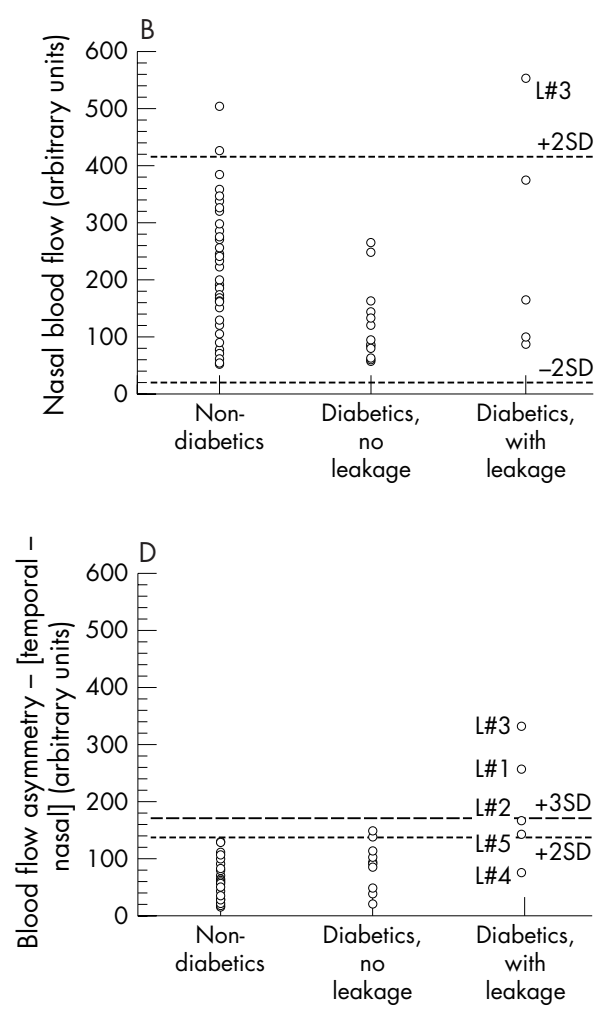

Figure 1 Scatter plots of macular capillary blood flow (arbitrary units) for normal subjects, diabetic patients without DMO and capillary leakage within the HRF scan area, and diabetic patients with DMO and capillary leakage within the SLDF scan area. (A) Foveal blood flow. (B) Nasal (relative to fovea) blood flow. (C) Temporal blood flow. (D) Relative nasal-temporal asymmetry of macular capillary blood flow. The broken lines show plus or minus 2 SD of the normal group mean nasal macular capillary blood flow value (and plus 3 SD in the case of nasal-temporal asymmetry of macular capillary blood flow). 
exhibited temporal macular capillary blood flow values that were lower than the normal 95\% confidence limits (that is, L\#5) - the patient exhibited DMO within the temporal area of the scan.

Four of the five patients with clinically significant DMO within the HRF scan area exhibited a nasal-temporal asymmetry of macular capillary blood flow values that were higher than the normal 95\% confidence limits (that is, L\#l, L\#2, L\#3, and L\#5). The other patient with clinically significant DMO within the scan area exhibited a relative nasal-temporal asymmetry of macular capillary blood flow values that were within the normal 95\% confidence limits (that is, L\#4) - the patient exhibited DMO within both the temporal and nasal scan areas. For all five patients with DMO within the HRF scan area, the lower value of macular capillary blood flow contributing to the nasal-temporal asymmetry was always to the side of the fovea exhibiting DMO and capillary leakage (or a greater extent of DMO and capillary leakage in the case of L\#4). Three of the 13 patients without clinically significant DMO within the scanned area (as indicated by stereo fundus biomicroscopy and confirmed by two medical retina specialists) exhibited a nasal-temporal asymmetry of macular capillary blood flow values that were higher than the normal 95\% confidence limits.

\section{DISCUSSION}

In this cohort of patients with clinically significant DMO, temporal macular capillary blood flow was found to be significantly lower than that of age matched non-diabetic subjects and nasal-temporal asymmetry of macular capillary blood flow was significantly higher. Interestingly, temporal macular capillary blood flow was not significantly different between the patients with and without DMO and capillary leakage within the scan area whereas nasal-temporal asymmetry of macular capillary blood flow was significantly higher for the patients with DMO and capillary leakage within the scan area. For the five patients exhibiting DMO within the scanned area, the lower value of macular capillary blood flow was always to the side of the fovea exhibiting DMO and capillary leakage (or the greater extent of DMO and capillary leakage in the case of L\#4). Macular capillary blood flow showed substantial inter-individual variation both for the non-diabetic subject group and for the clinically significant DMO group; the standard deviation of macular capillary blood flow was approximately $50 \%$ of the group mean. The influence of the foveal avascular zone (FAZ), which is devoid of retinal capillaries, was probably reflected by the trend for group mean foveal macular capillary blood flow to be lower than either temporal or nasal blood flow.

Using a video FFA technique, Arend and co-workers ${ }^{20}$ have previously reported that capillary blood velocity was significantly reduced in diabetic patients with cystoid macular oedema compared to non-diabetic subjects. This study also found that there was little difference in capillary blood velocity between diabetic patients with and without cystoid macular oedema. In a cross sectional study, Cuypers and coworkers ${ }^{21}$ found that retinal capillary blood flow measured by SLDF was associated with the level of diabetic retinopathy in the perifoveal macula area and approximately $7^{\circ}$ nasal to the disc. Blood flow was reduced for patients with proliferative retinopathy in comparison with patients with pre-proliferative, or non-proliferative, retinopathy. Interestingly, blood flow was found to be reduced in patients with exudative maculopathy in comparison with patients with no, preproliferative, or proliferative, diabetic retinopathy. Rawji and Flanagan ${ }^{22}$ recently reported the magnitude of intraocular and interocular asymmetry of SLDF derived retinal blood flow parameters in clinically normal volunteers. As anticipated, capillary perfusion was found to increase with eccentricity from the fovea and no significant interocular asymmetry was observed.

The relevance of the finding that temporal macular capillary blood flow was found to be significantly lower in patients with clinically significant DMO than that of age matched non-diabetic subjects has to be considered alongside the substantial inter-individual variation of the SLDF technique. In addition, there was a trend for the foveal and nasal capillary blood flow values to be lower but this did not reach statistical significance. Despite previous reports of a non-uniform distribution of diabetic vascular lesions, ${ }^{23}{ }^{24}$ the significantly lower temporal macular capillary blood flow can most likely be attributed to the lower inter-individual variability of the SLDF data (when compared to the group mean effect) at the temporal measurement site (table 1) rather than any particular anatomical or pathophysiological influence. Interestingly, three patients without clinically significant DMO within the scanned area exhibited an exaggerated relative nasal-temporal asymmetry of macular capillary blood flow (fig 1); whether these patients will ultimately develop clinically significant DMO within the scanned area is not known at this time.

Tsang and co-workers ${ }^{25}$ found artefactually high SLDF blood flow values to occur as a result of reduced brightness. However, the retina exhibits a localised reduction of reflectance intensity in areas of DMO. ${ }^{26}$ If the "brightness artefact" impacted upon the results of this study, macular capillary blood flow would be relatively higher in areas of DMO. Importantly, the finding that macular capillary blood flow was lower in areas of FFA leakage provides evidence of either a localised, rather than global, regulatory mechanism controlling macular capillary blood flow in patients with clinically significant DMO, or a local breakdown of this regulatory mechanism. The finding of a reduced blood flow in areas of FFA leakage is in broad agreement with the studies of Arend and co-workers ${ }^{20}$ and Cuypers and co-workers. ${ }^{21}$

In summary, this study demonstrated that SLDF derived measurements of temporal macular capillary blood flow were significantly lower in patients with clinically significant DMO than that of age matched non-diabetic subjects and that the relative nasal-temporal asymmetry of macular capillary blood flow was significantly exaggerated in patients with DMO. In addition, nasal-temporal asymmetry of macular capillary blood flow was significantly higher for the patients with DMO and capillary leakage within the scan area than for those without. Furthermore, capillary blood flow was reduced in areas of FFA leakage suggesting the presence of a localised regulatory mechanism controlling macular capillary blood flow in this patient group, or a local breakdown of this regulatory mechanism. Alternatively, the build up of extravascular fluid around leaking capillaries may be constrained, thus secondarily compromising capillary blood flow.

\section{ACKNOWLEDGEMENTS}

This work was funded by a postdoctoral fellowship grant awarded to Dr Hudson from the British Diabetic Association (now Diabetes UK). We thank Dr Georg Michelson for providing the AFFPIA software. We thank Erin Harvey, Department of Statistics and Actuarial Science, University of Waterloo for statistical advice and expertise.

\section{Authors' affiliations}

C Hudson, J G Flanagan, G S Turner, H C Chen, D McLeod, University Department of Ophthalmology, Royal Eye Hospital, Manchester M13 9WH, UK

C Hudson, J G Flanagan, M H Rawji, School of Optometry, University of Waterloo, Waterloo, Ontario N2L 3G1, Canada and Department of Ophthalmology, University of Toronto, Toronto, Ontario M5T 2S8, Canada 
Correspondence to: Chris Hudson, PhD, School of Optometry, University of Waterloo, 200 University Avenue East, Waterloo, Ontario N2L 3G1, Canada; chudson@sciborg.uwaterloo.ca

Accepted for publication 8 July 2004

\section{REFERENCES}

1 Kohner EM, Patel V, Rassam SMB. Role of blood flow and impaired autoregulation in the pathogenesis of diabetic retinopathy. Diabetes 1995:44:603-7.

2 Feke GT, Goger DG, Tagawa, et al. Laser Doppler technique for absolute measurement of blood speed in retinal vessels. IEEE Trans Biomed Eng 1987; $34: 673-80$

3 Riva CE, Grunwald JE, Petrig BL. Laser Doppler measurement of retinal blood velocity: validity of the single scattering model. Appl Opt 1985;24:605-11.

4 Riva CE, Harino S, Petrig BL, et al. Laser Doppler flowmetry in the optic nerve. Exp Eye Res 1992;55:499-504.

5 Michelson G, Schmauss B. Two dimensional mapping of the perfusion of the retina and optic nerve head. $\mathrm{Br} J$ Ophthalmol 1995;79:1126-32.

6 Michelson G, Langhans MJ, Groh MJM. Clinical investigation of the combination of a scanning laser ophthalmoscope and laser Doppler flowmeter. Ger J Ophthalmol 1995;4:342-9.

7 Michelson G, Schmauss B, Langhans MJ, et al. Principle, validity, and reliability of scanning laser Doppler flowmetry. J Glaucoma 1996:5:99-105.

8 Hafez AS, Bizzarro RLG, Rivard M, et al. Changes in optic nerve head blood flow after therapeutic intraocular pressure reduction in glaucoma patients and ocular hypertensives. Ophthalmology 2003;110:201-10.

9 Konno S, Feke GT, Yoshida A, et al. Retina blood flow changes in type 1 diabetes. A long-term, follow-up study. Invest Ophthalmol Vis Sci 1996;37:1 140-8.

10 Arend $\mathrm{O}$, Wolf $S$, Jung $F$, et al. Retinal microcirculation in patients with diabetes mellitus: Dynamic and morphological analysis of perifoveal capillary network. Br J Ophthalmol 1991;75:514-18.

11 Feke GT, Bizney SM, Ogasawara H, et al. Retinal circulatory abnormalities in type 1 diabetes. Invest Ophthalmol Vis Sci 1994;35:2968-75.
12 Sinclair SH. Macular retinal capillary hemodynamics in diabetic patients. Ophthalmology 1991; 98:1580-6.

13 Patel V, Rassam S, Newsom R, et al. Retinal blood flow in diabetic retinopathy. BMJ 1992;305:678-83

14 Grunwald JE, Brucker AJ, Schwartz SS, et al. Diabetic glycemic control and retinal blood flow. Diabetes 1990;39:602-7.

15 Klein R, Moss SE, Klein BEK, et al. The Wisconsin epidemio-logic study of diabetic retinopathy. IX. The incidence of macular oedema. Ophthalmology 1989;96:1501-10.

16 Early Treatment Diabetic Retinopathy Study Research Group. Treatment techniques and clinical guidelines for photocoagulation of diabetic macular edema. ETDRS Report Number 2. Ophthalmology 1987;94:761-74.

17 Chylack LT, Wolfe JK, Singer DM, et al. The lens opacities classification system III. Arch Ophthalmol 1993;111:831-6.

18 Schmetterer L, Woltz M. Review. Ocular blood flow and associated functional deviations in diabetic retinopathy. Diabetologia 1999;42:387-405.

19 Michelson G, Welzenbach J, Pal I, et al. Automatic full field analysis of perfusion images gained by scanning laser Doppler flowmetry. Br J Ophthalmol 1998;82:1294-300.

20 Arend O, Remky A, Harris A, et al. Macular microcirculation in cystoid maculopathy of diabetic patients. Br J Ophthalmol 1995;79:628-32.

21 Cuypers MHM, Kasanardjo JS, Polak BCP. Retinal blood flow changes in diabetic retinopathy measured with the Heidelberg scanning laser Doppler flowmeter. Graefes Arch Clin Exp Ophthalmol 2000;238:935-41.

22 Rawii MH, Flanagan JG. Intraocular and interocular symmetry in normal retinal capillary perfusion. J Glaucoma $2001 ; 10: 4-12$

23 Kern TS, Engerman RL. Vascular lesions in diabetes are distributed nonuniformly within the retina. Exp Eye Res 1995;60:545-9.

24 Bek T, Helgesen A. The regional distribution of diabetic retinopathy lesions may reflect risk factors for progression of the disease. Acta Ophthalmol Scand 2001;79:501-5.

25 Tsang AC, Harris A, Kagemann L, et al. Brightness alters Heidelberg Retinal Flowmeter measurement in an in vitro model. Invest Ophthalmol Vis Sci 1999;40:795-9.

26 Hudson C, Flanagan JG, Turner GS, et al. Scanning laser tomography Zprofile signal width as an objective index of retinal thickening due to macular oedema. Br J Ophthalmol 1998;82:121-30. 